\title{
Special Issue on Elderly Care Robotics - Technology and Ethics
}

\author{
Jim Torresen ${ }^{1} \cdot$ Ryo Kurazume ${ }^{2} \cdot$ Edson Prestes $^{3}$
}

Published online: 24 January 2020

(C) Springer Nature B.V. 2020

The number of older people living at home is increasing, and this trend is expected to continue in the coming years. In countries like Japan, the number of older people is beginning to surpass the number of young people due to the low birth rate and long life expectancy. Thus, there is a need for technology, in terms of software and hardware, that can support the elderly in independent living. A part of this is to help their family as well as make caregivers work effectively.

A number of mobile robot companions have been developed, providing advanced sensing, reasoning and control. However, although the available technology seems promising, it poses several challenges when used in real scenarios that range from how to handle complex and different environments to adequately address possible ethical issues that can arise from its use. For instance, technology can easily be seen as a threat to privacy, daily interpersonal contact and citizens' control over their own lives. It can even give rise to the elderly's feeling of being treated like an object rather than a human. Thus, it is important to address how robots and systems are going to be designed and used to avoid any negative impact and improve the elderly's quality of life. In addition to robot control and mechanics, ambient sensing and humanrobot interaction are important topics in research on elderly care technology. This special issue is organised complementary to the ICRA2018 Workshop on Elderly Care Robotics -

Jim Torresen

jimtoer@ifi.uio.no

Ryo Kurazume

kurazume@ ait.kyushu-u.ac.jp

Edson Prestes

prestes@inf.ufrgs.br

1 University of Oslo, Oslo, Norway

2 Kyushu University, Fukuoka, Japan

3 Federal University of Rio Grande do Sul (UFRGS), Porto Alegre, Brazil
Technology and Ethics (WELCARO), where authors of selected papers were invited to submit extended versions for this Journal of Intelligent \& Robotic Systems special issue.

The aim of the ICRA2018 workshop and this special issue is to provide a forum to disseminate and discuss recent and significant research within elderly care robotics related to both technology and ethics. The submitted papers have undergone peer review with one or two revised versions being checked by the initial reviewers. This has resulted in eight papers being accepted and presented in this special issue. We think they present promising state-of-the-art technology and relevant ethical considerations within elderly care robotics research. The first four papers deal with mobile robot companion design and control and are followed by three papers with a primary focus on human sensing. The final paper is about robot development standardization with regard to ethics.

The first paper in the special issue is by Sukhan Lee and Ahmed M.Naguib and presents the design and implementation of a new elderly care robot, named HomeMate. The following five service scenarios are designed: infotainment, video chatting, game playing, medicine alarm and, in particular, errand services. The strengths of the robot with respect to its sociability and dependability are demonstrated through the reported user studies.

William K. Juel et al. outline, in the second paper, the design of a novel welfare robot being developed in a project called SMOOTH. This paper describes the development of a concept for a novel welfare robot that is modular and affordable, which targets three use cases in a care center. The development is based on a participatory design process and by taking the strengths and limitations of selected, commercially available robots into account.

In the third paper, Conor McGinn et al. present Stevie - a socially assistive robot developed through the application of a Design-Thinking approach. Using a range of design techniques that have seldom been applied in a robotics context, a prototype has been designed, fabricated and evaluated using a mixed-methods approach. Experiments have been performed with four distinct user groups to explore the first impressions 
that the robot created, its perceived usefulness, and the potential for acceptance of the platform.

In the fourth paper, Caio Mucchiani et al. explore a lowcost mobile manipulation service robot for eldercare. That is, the paper identifies tasks an affordable mobile manipulator service robot could do to benefit older adults' independence in a supportive apartment living facility. This is undertaken by adapting a novel, low-cost telescopic robotic arm to a mobile base, with aspects of human-robot interaction investigated through a physical interactive game with the older adults.

The proposed technology in the fifth paper by Norihisa Paul Miyake et al. is a bedside agent for preventing falls. The system monitors a person's posture and behavior, and when it senses risk, it activates simultaneously a nurse call alarm and a voice call to the person to ensure that the person stays on the bed until a caregiver arrives, thus, reducing the chance of a fall. This paper describes the user-oriented design process of the agent system.

Serhan Cosar and Nicola Bellotto demonstrate in the sixth paper human re-identification with a thermal camera for a service robot. Being able to re-identify a person is important for service robots to allow them to perform personalized tasks and human-robot interactions. The paper outlines a system that can work using only a small amount of data. It includes various situations of human motion, poses and occlusion, and the data set is made publicly available for research purposes.

Atsushi Nakazawa et al. describe in the seventh papers a wearable person video analysis system for evaluating the skill levels of caregivers, as a part of a project that aims to quantize and analyze the tender-care technique known as Humanitude. The camera is worn by the caregiver and observes the face and eyes of the person receiving care. Results from eye contact detection experiments are reported in the paper.
Joanna Isabelle Olszewska et al. propose, in the final paper, a development methodology to support the robot standardization effort led by international, technical, and professional associations such as the Institute of Electrical and Electronics Engineers (IEEE). The proposed standard development life cycle is an iterative, collaborative, and incremental approach the authors have successfully applied to the development of the new IEEE Ontological Standard for Ethically Driven Robotics and Automation Systems (IEEE P7007 Standard).

The organising of the ICRA2018 workshop and this special issue is partially supported by the following research projects:

- JST CREST "Computational and cognitive neuroscientific approaches for understanding the tender care", under grant agreement JPMJCR17A5.

- MECS "Multimodal Elderly Care Systems (MECS)" funded by Research Council of Norway, under grant agreement 247,697

- COINMAC "Collaboration on Intelligent Machines" funded by Research Council of Norway, under grant agreement 261,645.

The guest editors would like to thank the reviewers for their efforts in reviewing the papers and the authors of the selected papers for their positive response to the reviewer's comments and suggestions. Also, special thanks are given to Editor-in-Chief Prof. Kimon P. Valavanis and Journals Editorial Office assistant Marie Veth Chua for their support of this special issue. We hope you will find the selected papers of interest, and that they are representing a valuable contribution to the elderly care robotics research domain. 